Wright State University

From the SelectedWorks of Nikolai V. Priezjev

Summer August 9, 2021

\title{
Accelerated rejuvenation in metallic glasses subjected to elastostatic compression along alternating directions
}

Nikolai Priezjev, Wright State University 


\title{
Accelerated rejuvenation in metallic glasses subjected to elastostatic compression along alternating directions
}

\author{
Nikolai V. Priezjev a,b \\ ${ }^{a}$ Department of Mechanical and Materials Engineering, Wright State University, Dayton, OH 45435, USA \\ ${ }^{\mathrm{b}}$ National Research University Higher School of Economics, Moscow 101000, Russia
}

\section{A R T I C L E I N F O}

\section{Keywords:}

Metallic glasses

Elastostatic loading

Mechanical processing

Yield stress

Molecular dynamics simulations

\begin{abstract}
A B S T R A C T
The influence of static stress and alternating loading direction on the potential energy and mechanical properties of amorphous alloys is investigated using molecular dynamics simulations. The model glass is represented via a binary mixture which is first slowly annealed well below the glass transition temperature and then subjected to elastostatic loading either along a single direction or along two and three alternating directions. We find that at sufficiently large values of the static stress, the binary glass becomes rejuvenated via collective, irreversible rearrangements of atoms. Upon including additional orientation of the static stress in the loading protocol, the rejuvenation effect is amplified and the typical size of clusters of atoms with large nonaffine displacements increases. As a result of prolonged mechanical loading, the elastic modulus and the peak value of the stress overshoot during startup continuous compression become significantly reduced, especially for loading protocols with alternating stress orientation. These findings are important for the design of novel processing methods to improve mechanical properties of metallic glasses.
\end{abstract}

\section{Introduction}

The development of novel fabrication techniques and processing methods that suppress crystallization and oxidation of metallic glasses is important for various biomedical and structural applications [1]. Although metallic glasses are known to possess exceptionally high yield stress and relatively large elastic strain limit due to their amorphous structure, upon further increasing stress, the plastic deformation becomes sharply localized within narrow regions, called shear bands, leading to material failure [2]. Notably, it was recently demonstrated that upon triaxial compression at room temperature, metallic glasses can be rejuvenated and exhibit strain-hardening, which inhibits the formation of shear bands and catastrophic failure during uniaxial tension or compression tests $[3,4]$. In general, common methods to rejuvenate amorphous alloys and improve their plasticity include irradiation, cold rolling, high-pressure torsion, flash annealing [5-8], elastostatic loading [9-18], and more recently discovered, cryogenic thermal cycling [19-21]. It was originally shown that a particularly simple way to induce rejuvenation and improve mechanical properties of metallic glasses is to apply a static stress slightly below the macroscopic yield stress [9]. In particular, the prolonged static loading within the elastic range induces irreversible rearrangements of atoms in a disordered solid and relocates the system into a higher potential energy state with a lower density [14]. More recently, it was found using atomistic simulations that binary glasses undergo a relaxation-to-rejuvenation transition upon increasing uniaxial static stress at about half the glass transition temperature $[15,18]$. However, the influence of alternating loading direction, multiaxial constraint, and hydrostatic pressure and temperature during elastostatic loading of amorphous alloys on their energy states and mechanical properties remains largely unexplored.

During the last decade, a number of molecular dynamics simulation studies have been carried out to investigate the atomic structure and mechanical properties of amorphous materials subjected to stress- or strain-driven periodic deformation [22-45]. Interestingly, it was demonstrated that at zero temperature atomic trajectories in a disordered solid under low-amplitude oscillatory shear eventually fall into the so-called 'limit cycles' and become exactly repeatable after one or more shear cycles $[25,27]$. In the presence of thermal fluctuations, rapidly quenched glasses subjected to periodic shear strain below the yielding point gradually relax towards low energy states with nearly reversible dynamics, typically during thousands of cycles, in a process termed 'mechanical annealing' [33,34,39,41,45]. More recently, it was shown that at low temperatures, the relaxation process can be accelerated in periodically driven metallic glasses if the shear orientation is

E-mail address: nikolai.priezjev@wright.edu. 
alternated along two or three spatial directions [39]. It was later found that when an additional shear orientation is introduced in the loading protocol, the number of transient cycles before the yielding transition is reduced but the critical strain amplitude remains unchanged [43]. Despite this progress, however, the optimization of thermomechanical processing methods requires further investigation of the operating conditions and details of loading protocols in order to access a wider range of energy states and improved properties of amorphous alloys.

In this paper, the influence of the static stress and variable loading direction on the potential energy and mechanical properties of disordered solids is investigated using molecular dynamics simulations. We study the binary mixture slowly annealed below the glass transition temperature and then loaded statically ether along a single direction or along two and three spatial directions. It will be shown that with increasing static stress, the potential energy increases and the size of plastically deformed regions becomes larger. Remarkably, the effect of rejuvenation is enhanced when an additional orientation of the static stress is included in the loading protocol. Furthermore, the results of mechanical tests after elastostatic loading indicate that both the elastic modulus and yield stress are reduced in rejuvenated samples.

The rest of the paper is divided into three sections. The details of molecular dynamics simulations and the loading protocol are described in the next section. The time dependence of the potential energy and mechanical properties as well as the analysis of nonaffine displacements are presented in Section 3. The main results are briefly summarized in the last section.

\section{Molecular dynamics (MD) simulations}

In our study, the amorphous alloy is represented via the KobAndersen (KA) binary mixture of two types of atoms with strong nonadditive interaction that suppresses crystallization upon cooling below the glass transition temperature [46]. The parametrization of the KA model is similar to the one used by Weber and Stillinger to study the amorphous metal alloy $\mathrm{Ni}_{80} \mathrm{P}_{20}$ [47]. In the KA model, the atoms of types $\alpha, \beta=A, B$ interact via the Lennard-Jones (LJ) potential as follows:

$V_{\alpha \beta}(r)=4 \varepsilon_{\alpha \beta}\left[\left(\frac{\sigma_{\alpha \beta}}{r}\right)^{12}-\left(\frac{\sigma_{\alpha \beta}}{r}\right)^{6}\right]$,

with the standard parametrization for the energy and length scales: $\varepsilon_{A A}$ $=1.0, \varepsilon_{A B}=1.5, \varepsilon_{B B}=0.5, \sigma_{A A}=1.0, \sigma_{A B}=0.8, \sigma_{B B}=0.88$, and $m_{A}=$ $m_{B}$ [46]. To speed up the computation, the cutoff radius of the LJ potential is set to $r_{c, \alpha \beta}=2.5 \sigma_{\alpha \beta}$. The system consists of $N=60000$ atoms. Throughout the study, the physical quantities are reported using the LJ units of length, mass, energy, and time, as follows: $\sigma=\sigma_{A A}, m=m_{A}, \varepsilon=$ $\varepsilon_{A A}$, and, correspondingly, $\tau=\sigma \sqrt{m / \varepsilon}$. The MD simulations were carried out using the LAMMPS code [48] with the integration time step $\Delta t_{M D}=0.005 \tau[49]$.

The sample preparation procedure is similar to the one employed in the previous study on elastostatic loading along a single direction [18]. Specifically, the binary mixture was first equilibrated in a periodic box at the temperature $T_{L J}=1.0 \varepsilon / k_{B}$ and zero pressure. Here, $k_{B}$ denotes the Boltzmann constant. In all simulations, the temperature was regulated via the Nosé-Hoover thermostat $[48,49]$. After equilibration, the system was linearly cooled with the computationally slow rate $10^{-5} \varepsilon$ / $k_{B} \tau$ at zero pressure to the reference temperature of $0.01 \varepsilon / k_{B}$. In the previous study, the glass transition temperature of the KA model was found to be about $0.40 \varepsilon / k_{B}$, when the mixture was cooled with the rate $10^{-5} \varepsilon / k_{B} \tau$ at zero pressure [7].

The loading procedure includes heating the glass to the annealing temperature $T_{a}$ and at the same time increasing the normal stress during $5000 \tau$, then annealing the system during the time interval $t_{a}$ at a constant value of the normal stress, followed by subsequent relocation of the glass to the reference state $\left(T_{L J}=0.01 \varepsilon / k_{B}\right.$ and $\left.P=0\right)$ during $5000 \tau(e$. $g$., see Fig. 1). It was previously shown that rejuvenation is enhanced

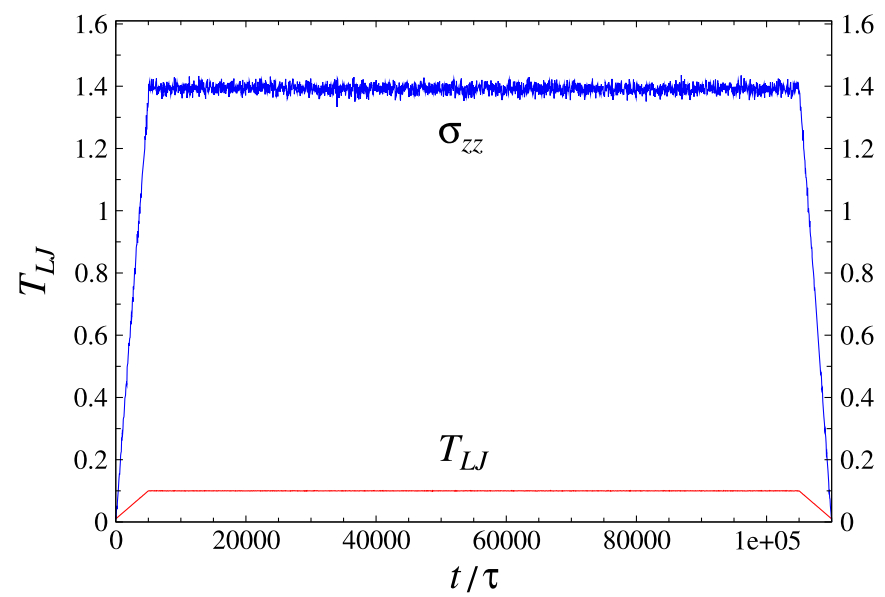

Fig. 1. (Color online) The temperature (left axis, red line) and normal stress (right axis, blue line) profiles during the loading protocol. The temperature varies linearly from $T_{L J}=0.01 \varepsilon / k_{B}$ to $T_{a}=0.1 \varepsilon / k_{B}$ during $5000 \tau$, remains $T_{a}=0.1 \varepsilon / k_{B}$ during the annealing time $t_{a}=10^{5} \tau$, and then reduces to $T_{L J}=$ $0.01 \varepsilon / k_{B}$. The variation of the normal stress is similar except that $\sigma_{z z}(0)=0$ and $\sigma_{z z}\left(1.1 \times 10^{5} \tau\right)=0$.

during elastostatic loading along a single direction at the annealing temperature $T_{a}=0.1 \varepsilon / k_{B}$ [18], and, therefore, in the present study all simulations were carried out at this value of $T_{a}$. Furthermore, the static stress was applied either along the same direction or alternated along two and three spatial directions. In all cases, the normal stress components perpendicular to the imposed static stress were set to zero. During production runs, the stress components, potential energy, atomic configurations, and system dimensions were stored at $T_{L J}=0.01 \varepsilon / k_{B}$ and $P=0$. The data were accumulated only for one realization of disorder due to computational limitations. For example, a typical run during $t_{a}=$ $2.4 \times 10^{6} \tau$ using 28 processors required about $360 \mathrm{~h}$ at the Ohio Supercomputer Cluster.

\section{Results}

It has long been realized that the rate at which a multicomponent mixture is cooled across the glass transition point is an important factor that determines the atomic structure and the potential energy state of a glass [2]. Hence, a more slowly annealed glass is settled at a deeper energy level, whereas rapidly quenched glasses typically have higher potential energy [50]. Moreover, depending on the amplitude of periodic strain deformation, the glass can be either rejuvenated or relaxed [51]. In the case of elastostatic loading, the glass can be rejuvenated via collective rearrangements of atoms, predominantly near soft spots, if the imposed stress is sufficiently large and the annealing temperature is well below the glass transition temperature [18]. Interestingly, a transition from relaxed to rejuvenated states upon increasing static stress at about half the glass transition temperature was reported in the recent MD study [18]. In principle, the potential energy can be further increased during elastostatic loading if the orientation of the static stress is occasionally changed, thus allowing different clusters of atoms to rearrange irreversibly. In the present study, we explore the effect of rejuvenation in a well annealed binary glass for three loading protocols; namely, when the static stress is applied along a single direction or changed along two or three spatial directions.

The results of the previous MD study on relaxation and rejuvenation of binary glasses subjected to elastostatic loading [18] provide guidance on the choice of parameters for the loading protocol with alternating orientation of the static stress. Thus, it was shown that rejuvenation is enhanced if the annealing temperature is sufficiently below the glass transition temperature $T_{g} \approx 0.40 \varepsilon / k_{B}$ at $P=0$ [18]. In the present study, all simulation results are reported for the annealing temperature 
$T_{L J}=0.1 \varepsilon / k_{B}$, at which the aging effects are negligible during $t_{a}=2.4 \times$ $10^{6} \tau$ [18]. Moreover, it was also found that during compression of a well annealed glass at the constant strain rate $\dot{\varepsilon}=10^{-5} \tau^{-1}$ at $T_{L J}=0.1 \varepsilon / k_{B}$, the peak value of the stress overshoot is $\sigma_{Y} \approx 1.9 \varepsilon \sigma^{-3}$ [18]. Therefore, the loading protocol in the present study includes four values of the static stress below $\sigma_{Y}$; namely, $0.5 \varepsilon \sigma^{-3}, 1.1 \varepsilon \sigma^{-3}, 1.3 \varepsilon \sigma^{-3}$, and $1.4 \varepsilon \sigma^{-3}$. Furthermore, the static stress was applied either along a single $(\widehat{z})$ direction or alternated along two $(\widehat{z}$ and $\widehat{x})$ and three $(\widehat{z}, \widehat{x}$, and $\widehat{y})$ directions. In the last two cases, the orientation of the static stress was changed every $10^{5} \tau$.

The dependence of the potential energy on the annealing time and loading protocol is shown in Fig. 2 for the indicated values of the static stress. Note that the data in Fig. 2 are taken at the reference state, $T_{L J}=$ $0.01 \varepsilon / k_{B}$ and $P=0$, whereas the static stress was applied at the temperature $T_{a}=0.1 \varepsilon / k_{B}$. As shown in Fig. 2, the glass becomes rejuvenated when the imposed static stress is sufficiently large (greater than $0.5 \varepsilon \sigma^{-3}$ ) and $t_{a} \lesssim 2.4 \times 10^{6} \tau$. Note that the increase in potential energy with respect to the dashed line is larger for higher values of the static stress. This is consistent with the results of the previous MD study on elastostatic loading where the static stress was applied along a single direction [18]. It can be clearly seen in Fig. 2 (c, d) that in the case of alternating orientation of the static stress, the effect of rejuvenation is significantly amplified. This trend can be rationalized by realizing that upon changing the orientation of the static stress, the stress in the system becomes redistributed and a different set of localized rearrangements can be facilitated (during the time interval $10^{5} \tau$ ), leading to faster rejuvenation. As shown in Fig. 2 (c, d), the largest increase in energy is attained when the static stress is alternated along all three spatial directions.

We further comment that during elastostatic loading at $1.4 \varepsilon \sigma^{-3}$ along alternating directions, the samples were extensively compressed along the direction of the applied stress. Therefore, the data are not reported for $t_{a} \gtrsim 1.2 \times 10^{6} \tau$ in Fig. 2 (d). For example, the time dependence of the potential energy and the system size for alternating loading at $1.4 \varepsilon \sigma^{-3}$ is presented in Fig. 3. It can be seen in Fig. 3 (a) that, following a gradual compression for $t_{a} \lesssim 1.26 \times 10^{6} \tau$, the system becomes significantly deformed during a relatively short time interval $\approx 2$ $\times 10^{3} \tau$. This plastic strain is unrecoverable, and the corresponding

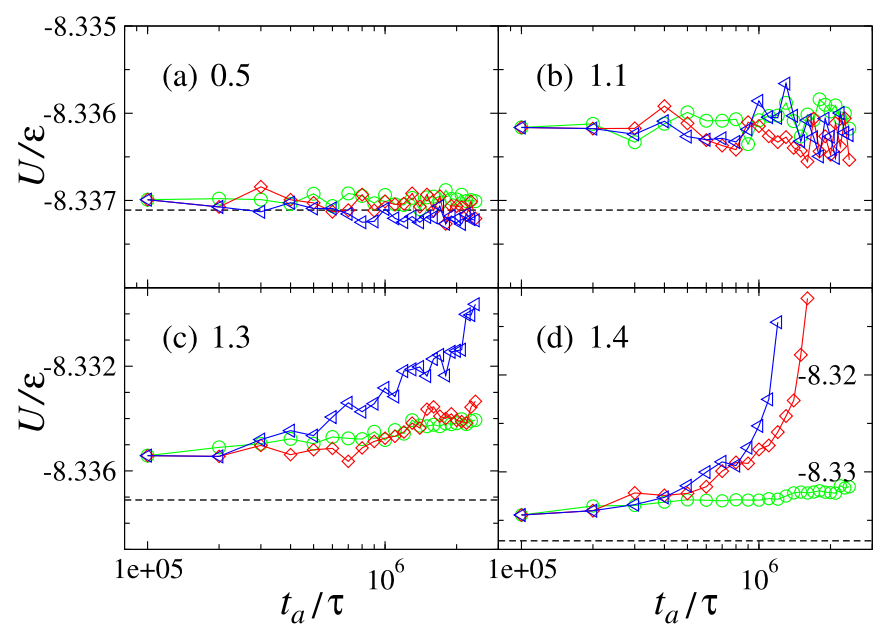

Fig. 2. (Color online) The potential energy per atom as a function of the annealing time, $t_{a} / \tau$, when the static stress is (a) $0.5 \varepsilon \sigma^{-3}$, (b) $1.1 \varepsilon \sigma^{-3}$, (c) $1.3 \varepsilon \sigma^{-3}$, and (d) $1.4 \varepsilon \sigma^{-3}$. The static stress is applied along the $\widehat{z}$ direction (green circles), alternated along the $\widehat{z}$ and $\widehat{x}$ directions (red diamonds), and alternated along the $\widehat{z}, \widehat{x}$, and $\widehat{y}$ directions (blue triangles). The horizontal dashed lines indicate the potential energy level $U \approx-8.337 \varepsilon$ before elastostatic loading was applied. (For interpretation of the references to colour in this figure legend, the reader is referred to the web version of this article.)

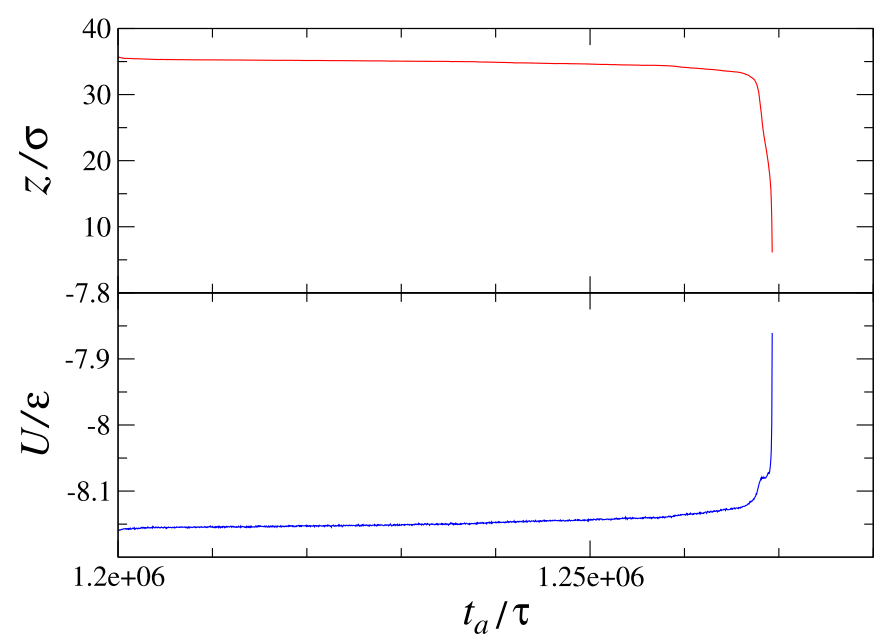

Fig. 3. (Color online) The time dependence of the system size along the $\widehat{z}$ axis (the upper panel) and the potential energy (the lower panel) when the static stress is $1.4 \varepsilon \sigma^{-3}$ and temperature is $T_{a}=0.1 \varepsilon / k_{B}$. The loading protocol consists of alternating static stress along all three spatial directions (see text for details) (For interpretation of the references to colour in this figure legend, the reader is referred to the web version of this article.).

change in the potential energy is shown in Fig. 3 (b). In general, we find that the loading time until plastic flow is reduced for larger values of the static stress and loading protocols with alternating stress orientation.

The spatial organization of stress-induced, irreversible rearrangements of atoms can be analyzed via their nonaffine displacements [52]. More specifically, the nonaffine measure for a particular atom can be computed by using the transformation matrix $\mathbf{J}_{i}$, which linearly transforms positions of neighboring atoms, and at the same time minimizes the following expression:

$D^{2}(t, \Delta t)=\frac{1}{N_{i}} \sum_{j=1}^{N_{i}}\left\{\mathbf{r}_{j}(t+\Delta t)-\mathbf{r}_{i}(t+\Delta t)-\mathbf{J}_{i}\left[\mathbf{r}_{j}(t)-\mathbf{r}_{i}(t)\right]\right\}^{2}$,

where $\Delta t$ is the time interval between two successive configurations and the sum is taken over the neighboring atoms within $1.5 \sigma$ from the position $\mathbf{r}_{i}(t)$. It was originally demonstrated by Falk and Langer that the nonaffine measure is an excellent indicator of irreversible shear transformations in deformed amorphous solids [52]. More recently, the appearance of clusters of atoms with relatively large nonaffine displacements was connected to rejuvenation of elastostatically loaded binary glasses [18]. In recent years, the collective rearrangements of atoms in disordered solids were also reported during steady [53-57] and periodic [28-35,39-41,43,45] deformation and thermal cycling [58-60].

The effects of loading time and stress orientation on plastic rearrangements of atoms are presented in Figs. 4-6. In all cases, the value of the static stress is fixed to $1.3 \varepsilon \sigma^{-3}$, and the lag time in Eq. (2) is set $\Delta t=$ $6 \times 10^{5} \tau$. The reference times $t=0,6 \times 10^{5} \tau, 1.2 \times 10^{6} \tau$, and $1.8 \times$ $10^{6} \tau$ are chosen within the rejuvenation regime, $0 \leqslant t_{a} \leqslant 2.4 \times 10^{6} \tau$, as shown in Fig. 2 (c). The loading protocol consists of the static stress applied either along a single ( $\widehat{z}$ ) direction (see Fig. 4) or alternated along two ( $\widehat{z}$ and $\widehat{x}$ ) directions (see Fig. 5) or all three directions (see Fig. 6). It is clearly seen in Figs. 4-6 that the size of clusters of atoms with large nonaffine displacements increases when an additional spatial dimension for the stress orientation is included in the loading protocol. Note also that upon loading along a single direction, a large fraction of the system deforms plastically during the first $\Delta t=6 \times 10^{5} \tau$, but only a few isolated clusters are present at longer times, as shown in Fig. 4. This observation correlates with a steep increase and subsequent slow growth of the potential energy, as indicated by green circles in Fig. 2 (c). By contrast, when the stress orientation is alternated along all three spatial 

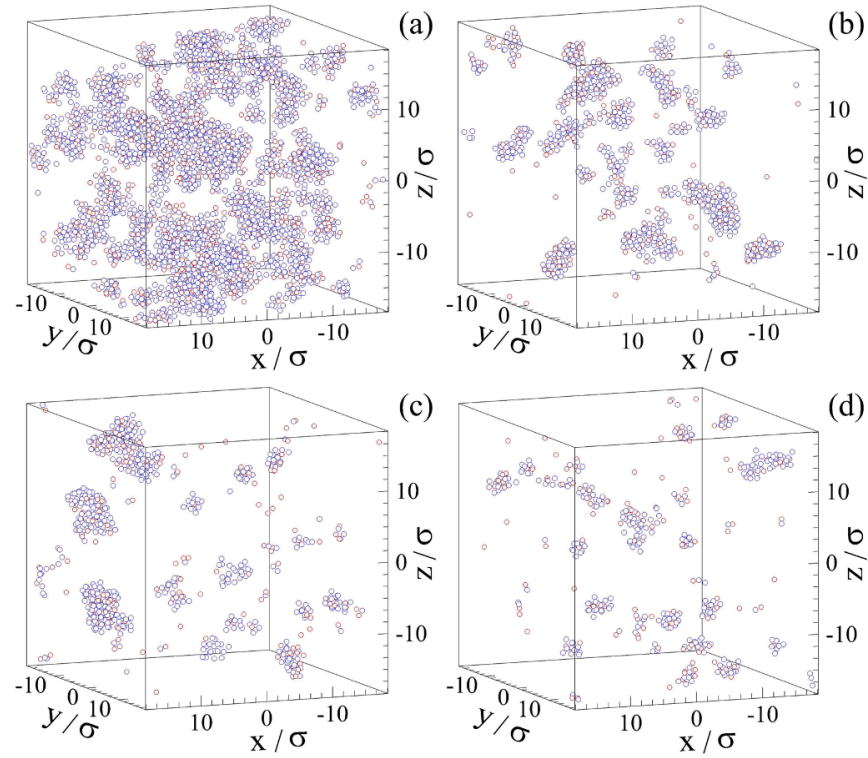

Fig. 4. (Color online) The snapshots of atomic positions with the nonaffine measure $D^{2}(t, \Delta t)$ : (a) $D^{2}\left(0,6 \times 10^{5} \tau\right)>0.04 \sigma^{2}$, (b) $D^{2}\left(6 \times 10^{5} \tau, 6 \times 10^{5} \tau\right)>$ $0.04 \sigma^{2}$, (c) $D^{2}\left(1.2 \times 10^{6} \tau, 6 \times 10^{5} \tau\right)>0.04 \sigma^{2}$, and (d) $D^{2}\left(1.8 \times 10^{6} \tau, 6 \times 10^{5} \tau\right)$ $>0.04 \sigma^{2}$. The static stress $1.3 \varepsilon \sigma^{-3}$ is applied along a single $(\widehat{z})$ direction. The potential energy for the same sample is indicated by green circles in Fig. 2 (c). (For interpretation of the references to colour in this figure legend, the reader is referred to the web version of this article.)
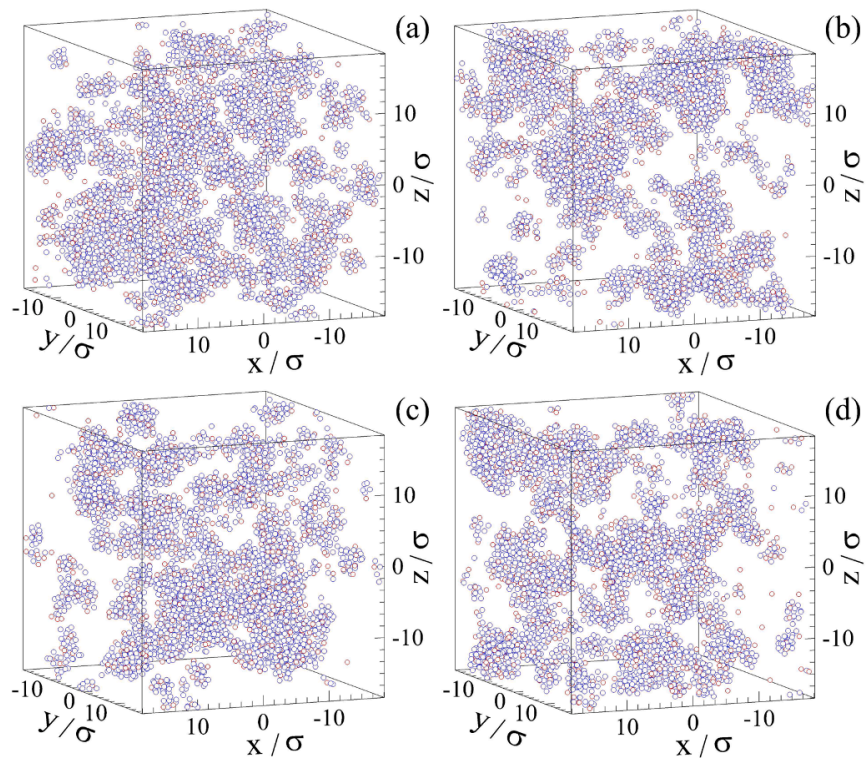

Fig. 5. (Color online) The atomic configurations with the nonaffine measure $D^{2}(t, \Delta t):$ (a) $D^{2}\left(0,6 \times 10^{5} \tau\right)>0.04 \sigma^{2}$, (b) $D^{2}\left(6 \times 10^{5} \tau, 6 \times 10^{5} \tau\right)>0.04 \sigma^{2}$, (c) $D^{2}\left(1.2 \times 10^{6} \tau, 6 \times 10^{5} \tau\right)>0.04 \sigma^{2}$, and (d) $D^{2}\left(1.8 \times 10^{6} \tau, 6 \times 10^{5} \tau\right)>0.04 \sigma^{2}$. The static stress $1.3 \varepsilon \sigma^{-3}$ is alternated along the $\widehat{z}$ and $\widehat{x}$ directions. The same data are denoted by red diamonds in Fig. 2 (c). (For interpretation of the references to colour in this figure legend, the reader is referred to the web version of this article.)

directions, most of the atoms undergo large nonaffine displacements (larger than the typical cage size $\approx 0.1 \sigma$ ), and the number of rearrangements increases with time (see Fig. 6), which corresponds to the enhanced rejuvenation shown by blue triangles in Fig. 2 (c).

We next present the elastic modulus, $E$, in Fig. 7 and the yield stress, $\sigma_{Y}$, in Fig. 8 for the subset of data points shown in Fig. 2. The mechanical
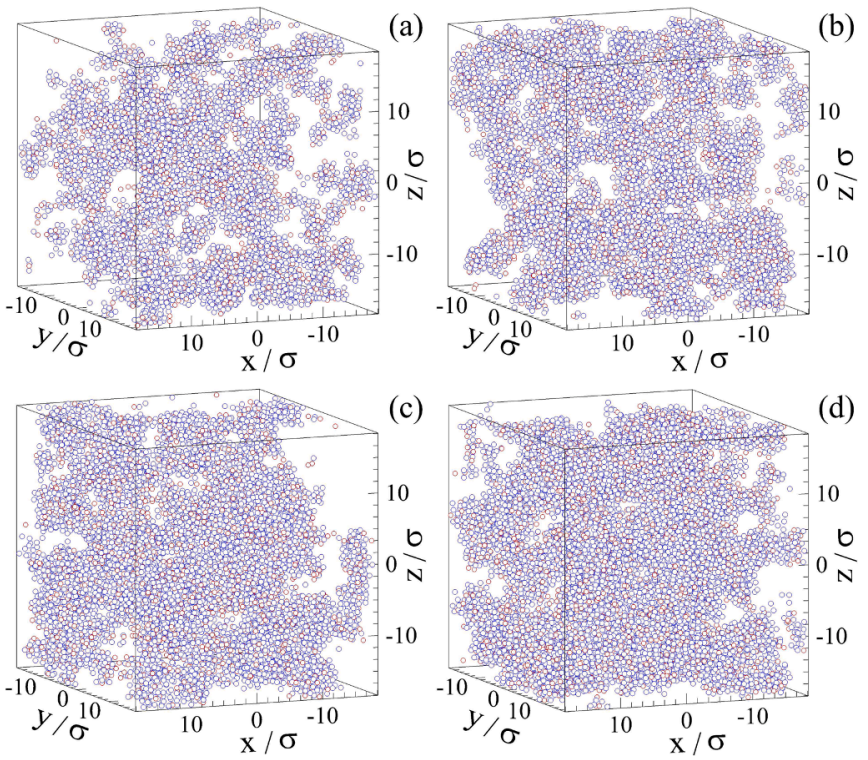

Fig. 6. (Color online) The positions of atoms with the nonaffine measure $D^{2}(t$ $\Delta t):$ (a) $D^{2}\left(0,6 \times 10^{5} \tau\right)>0.04 \sigma^{2}$, (b) $D^{2}\left(6 \times 10^{5} \tau, 6 \times 10^{5} \tau\right)>0.04 \sigma^{2}$, (c) $D^{2}\left(1.2 \times 10^{6} \tau, 6 \times 10^{5} \tau\right)>0.04 \sigma^{2}$, and (d) $D^{2}\left(1.8 \times 10^{6} \tau, 6 \times 10^{5} \tau\right)>0.04 \sigma^{2}$. The static stress $1.3 \varepsilon \sigma^{-3}$ is alternated along the $\widehat{z}, \widehat{x}$, and $\widehat{y}$ directions. The data are marked by blue triangles in Fig. 2 (c). (For interpretation of the references to colour in this figure legend, the reader is referred to the web version of this article.)

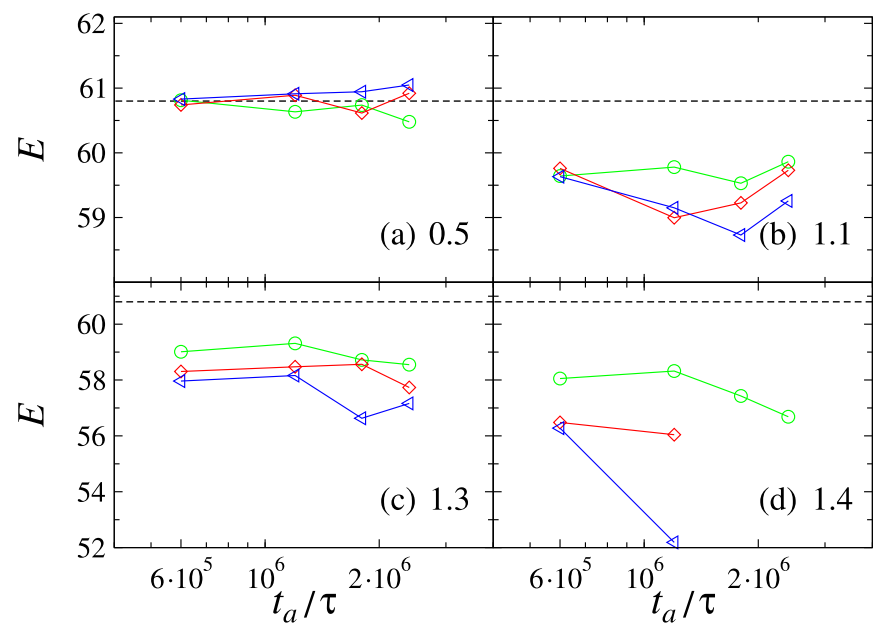

Fig. 7. (Color online) The variation of the elastic modulus $E$ (in units of $\varepsilon \sigma^{-3}$ ) as a function of the annealing time, $t_{a} / \tau$, for the values of the static stress (a) $0.5 \varepsilon \sigma^{-3}$, (b) $1.1 \varepsilon \sigma^{-3}$, (c) $1.3 \varepsilon \sigma^{-3}$, and (d) $1.4 \varepsilon \sigma^{-3}$. The elastostalic loading is applied along the $\widehat{z}$ direction (green circles), alternated along the $\widehat{z}$ and $\widehat{x}$ directions (red diamonds), and alternated along the $\widehat{z}, \widehat{x}$, and $\widehat{y}$ directions (blue triangles). The dashed black lines denote the elastic modulus $E=60.80 \varepsilon \sigma^{-3}$ at $T_{L J}=0.01 \varepsilon / k_{B}$ before elastostalic loading. Note that the vertical scales are different in the upper and lower panels. (For interpretation of the references to colour in this figure legend, the reader is referred to the web version of this article.)

properties reported in Figs. 7 and 8 were computed during startup continuous compression with the constant strain rate $\dot{\varepsilon}=10^{-5} \tau^{-1}$. More specifically, the elastic modulus was calculated from the best linear fit to the data at small strain, $\varepsilon \leqslant 0.01$, whereas the yield stress is defined by the peak value of the stress overshoot at about $5 \%$ strain. The samples were strained along the $\widehat{x}, \hat{y}$, and $\widehat{z}$ directions, and then the data for $E$ and $\sigma_{Y}$ were averaged over three spatial directions. The horizontal dashed lines 


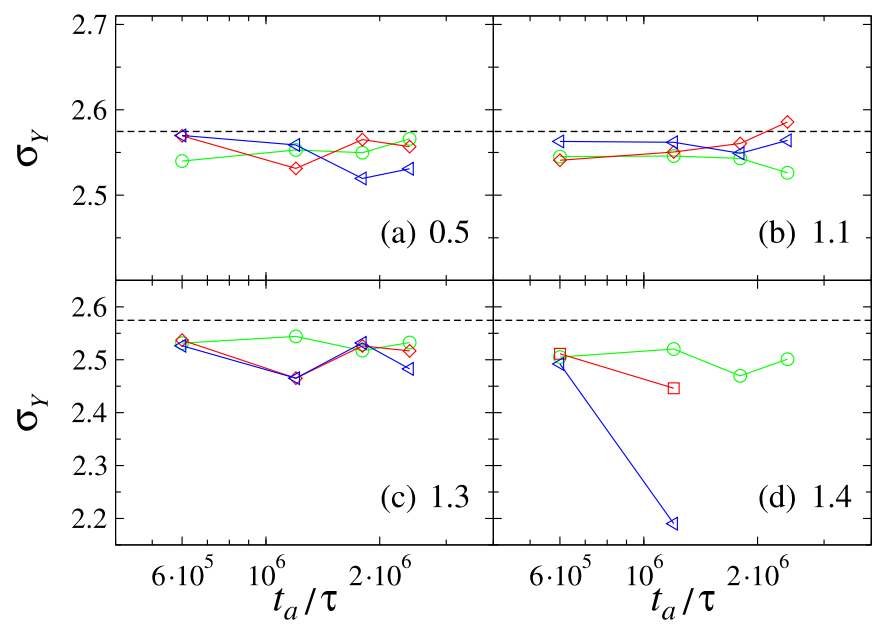

Fig. 8. (Color online) The yielding peak $\sigma_{Y}$ (in units of $\varepsilon \sigma^{-3}$ ) versus the annealing time for the static stress (a) $0.5 \varepsilon \sigma^{-3}$, (b) $1.1 \varepsilon \sigma^{-3}$, (c) $1.3 \varepsilon \sigma^{-3}$, and (d) $1.4 \varepsilon \sigma^{-3}$. The static stress is imposed along the $\widehat{z}$ direction (green circles), alternated along the $\widehat{z}$ and $\widehat{x}$ directions (red diamonds), and alternated along the $\widehat{z}, \widehat{x}$, and $\widehat{y}$ directions (blue triangles). The dashed lines indicate the peak value of the stress overshoot $\sigma_{Y}=2.57 \varepsilon \sigma^{-3}$ before static loading. (For interpretation of the references to colour in this figure legend, the reader is referred to the web version of this article.)

in Figs. 7 and 8 indicate the values of $E$ and $\sigma_{Y}$ before elastostatic loading was applied. As expected, the general trend for the time dependence of $E$ and $\sigma_{Y}$ is inversely correlated with the variation of the potential energy shown in Fig. 2. In particular, it can be observed that the yield stress is only slightly reduced for the static loading at $0.5 \varepsilon \sigma^{-3}$ and $1.1 \varepsilon \sigma^{-3}$, while the effect of alternating loading becomes significant at larger values of the static stress, $1.3 \varepsilon \sigma^{-3}$ and $1.4 \varepsilon \sigma^{-3}$ (see Fig. 8). Thus, the maximum decrease of the yield stress due to elastostatic loading at $1.4 \varepsilon \sigma^{-3}$ along three directions is about $15 \%$ at $t=1.2 \times 10^{6} \tau$, as shown in Fig. 8 (c). Altogether, these results demonstrate that both elastic modulus and yield stress become reduced upon loading at sufficiently large values of the static stress; and the effect is amplified if the loading orientation is periodically alternated along two or three spatial directions.

Finally, it should be commented that the simulation results reported in the present study are based on the LJ potential in the KA model, which might not accurately describe the atomic structure in the glass phase (e. $g$., the icosahedral short-range order) and inherently unstable to crystallization at sufficiently long timescales and large system sizes [61]. More recently, a modified LJ potential with the bump term was used to study the mechanical properties of metallic glasses with controllable intrinsic ductility [62]. In the future, it will be instructive to carry out MD simulations with more realistic pairwise potentials in order to verify that the effect of accelerated rejuvenation due to alternating loading can be generalized to other typical metallic glass systems.

\section{Conclusions}

In summary, we investigated the effects of elastostatic loading and alternating orientation of the static stress on the potential energy states and mechanical properties of amorphous alloys using molecular dynamics simulations. The well annealed alloy was prepared by slowly cooling a binary mixture at zero pressure from the liquid state to a temperature well below the glass transition temperature. After annealing, the binary glass was subjected to prolonged elastostatic compression either along a single direction or along two and three alternating directions. It was demonstrated that elastostatic loading at sufficiently large values of stress induces collective plastic events and significant rejuvenation. Moreover, upon introducing an alternating stress orientation in the loading protocol, the potential energy is further increased with respect to the untreated sample, and the typical size of plastic rearrangements becomes comparable with the system size. Finally, the mechanical properties were probed by continuously compressing the treated samples at a constant strain rate. We found that both the elastic modulus and the peak value of the stress overshoot are reduced with increasing loading time or alternating stress orientation [48].

\section{Declaration of Competing Interest}

The authors declare that they have no known competing financial interests or personal relationships that could have appeared to influence the work reported in this paper.

\section{Acknowledgments}

Financial support from the National Science Foundation (CNS1531923) and the ACS Petroleum Research Fund (60092-ND9) is gratefully acknowledged. The article was prepared within the framework of the HSE University Basic Research Program and funded in part by the Russian Academic Excellence Project '5-100'. The molecular dynamics simulations were performed using the LAMMPS open-source code developed at Sandia National Laboratories [48]. The numerical simulations were carried out at the Wright State University's Computing Facility and the Ohio Supercomputer Center.

\section{References}

[1] Z. Li, Z. Huang, F. Sun, X. Li, J. Ma, Forming of metallic glasses: mechanisms and processes, Mater. Today Adv. 7 (2020) 100077.

[2] Y. Sun, A. Concustell, A.L. Greer, Thermomechanical processing of metallic glasses: extending the range of the glassy state, Nat. Rev. Mater. 1 (2016) 16039.

[3] J. Pan, Y.P. Ivanov, W.H. Zhou, Y. Li, A.L. Greer, Strain-hardening and suppression of shear-banding in rejuvenated bulk metallic glass, Nature 578 (2020) 559.

[4] A.D. Phan, A. Zaccone, V.D. Lam, K. Wakabayashi, Theory of pressure-induced rejuvenation and strain-hardening in metallic glasses, 2020, arXiv:2007.15524.

[5] M. Wakeda, J. Saida, J. Li, S. Ogata, Controlled rejuvenation of amorphous metals with thermal processing, Sci. Rep. 5 (2015) 10545.

[6] S. Kuchemann, P.M. Derlet, C. Liu, D. Rosenthal, G. Sparks, W.S. Larson, R. Maass, Energy storage in metallic glasses via flash annealing, Adv. Funct. Mater. 28 (2018) 1805385.

[7] N.V. Priezjev, Atomistic modeling of heat treatment processes for tuning the mechanical properties of disordered solids, J. Non-Cryst. Solids 518 (2019) 128.

[8] M. Wang, H. Liu, J. Li, Q. Jiang, W. Yang, C. Tang, Thermal-pressure treatment for tuning the atomic structure of metallic glass Cu-Zr, J. Non-Cryst. Solids 535 (2020) 119963.

[9] K.-W. Park, C.-M. Lee, M. Wakeda, Y. Shibutani, M.L. Falk, J.C. Lee, Elastostatically induced structural disordering in amorphous alloys, Acta Mater. 56 (2008) 5440.

[10] C.-M. Lee, K.-W. Park, B.-J. Lee, Y. Shibutani, J.C. Lee, Structural disordering of amorphous alloys: amolecular dynamics analysis, Scr. Mater. 61 (2009) 911.

[11] Y. Tong, W. Dmowski, Y. Yokoyama, G. Wang, P.K. Liaw, T. Egami, Recovering compressive plasticity of bulk metallic glasses by high-temperature creep, Scr. Mater. 69 (2013) 570.

[12] Y.M. Wang, M. Zhang, L. Liu, Mechanical annealing in the homogeneous deformation of bulk metallic glass under elastostatic compression, Scr. Mater. 102 (2015) 67.

[13] L.Z. Zhao, R.J. Xue, Y.Z. Li, W.H. Wang, H.Y. Bai, Revealing localized plastic flow in apparent elastic region before yielding in metallic glasses, J. Appl. Phys. 118 (2015) 244901.

[14] A.L. Greer, Y.H. Sun, Stored energy in metallic glasses due to strains within the elastic limit, Philos. Mag. 96 (2016) 1643.

[15] M. Zhang, Y.M. Wang, F.X. Li, S.Q. Jiang, M.Z. Li, L. Liu, Mechanical relaxation-torejuvenation transition in a Zr-based bulk metallic glass, Sci. Rep. 7 (2017) 625.

[16] J. Pan, Y.X. Wang, Q. Guo, D. Zhang, A.L. Greer, Y. Li, Extreme rejuvenation and softening in a bulk metallic glass, Nat. Commun. 9 (2018) 560.

[17] M. Samavatian, R. Gholamipour, A.A. Amadeh, S. Mirdamadi, Role of tensile elastostatic loading on atomic structure and mechanical properties of $\mathrm{Zr}_{55} \mathrm{Cu}_{30} \mathrm{Ni}_{5} \mathrm{Al}_{10}$ bulk metallic glass, Mater. Sci. Eng. A 753 (2019) 218.

[18] N.V. Priezjev, Aging and rejuvenation during elastostatic loading of amorphous alloys: a molecular dynamics simulation study, Comput. Mater. Sci. 168 (2019) 125.

[19] S.V. Ketov, Y.H. Sun, S. Nachum, Z. Lu, A. Checchi, A.R. Beraldin, H.Y. Bai, W. H. Wang, D.V. Louzguine-Luzgin, M.A. Carpenter, A.L. Greer, Rejuvenation of metallic glasses by non-affine thermal strain, Nature 524 (2015) 200.

[20] W. Guo, J. Saida, M. Zhao, S. Lu, S. Wu, Rejuvenation of Zr -based bulk metallic glass matrix composite upon deep cryogenic cycling, Mater. Lett. 247 (2019) 135. 
[21] M. Samavatian, R. Gholamipour, A.A. Amadeh, S. Mirdamadi, Correlation between plasticity and atomic structure evolution of a rejuvenated bulk metallic glass, Metall. Mater. Trans. A 50 (2019) 4743.

[22] Y.C. Lo, H.S. Chou, Y.T. Cheng, J.C. Huang, J.R. Morris, P.K. Liaw, Structural relaxation and self-repair behavior in nano-scaled $\mathrm{Zr}$-Cu metallic glass under cyclic loading: molecular dynamics simulations, Intermetallics 18 (2010) 954.

[23] N.V. Priezjev, Heterogeneous relaxation dynamics in amorphous materials under cyclic loading, Phys. Rev. E 87 (2013) 052302.

[24] D. Fiocco, G. Foffi, S. Sastry, Oscillatory athermal quasistatic deformation of a model glass, Phys. Rev. E 88 (2013) 020301(R).

[25] I. Regev, T. Lookman, C. Reichhardt, Onset of irreversibility and chaos in amorphous solids under periodic shear, Phys. Rev. E 88 (2013) 062401.

[26] N.V. Priezjev, Dynamical heterogeneity in periodically deformed polymer glasses, Phys. Rev. E 89 (2014) 012601.

[27] I. Regev, J. Weber, C. Reichhardt, K.A. Dahmen, T. Lookman, Reversibility and criticality in amorphous solids, Nat. Commun. 6 (2015) 8805

[28] N.V. Priezjev, Reversible plastic events during oscillatory deformation of amorphous solids, Phys. Rev. E 93 (2016) 013001.

[29] N.V. Priezjev, Nonaffine rearrangements of atoms in deformed and quiescent binary glasses, Phys. Rev. E 94 (2016) 023004.

[30] T. Kawasaki, L. Berthier, Macroscopic yielding in jammed solids is accompanied by a non-equilibrium first-order transition in particle trajectories, Phys. Rev. E 94 (2016) 022615.

[31] P. Leishangthem, A.D.S. Parmar, S. Sastry, The yielding transition in amorphous solids under oscillatory shear deformation, Nat. Commun. 8 (2017) 14653.

[32] N.V. Priezjev, Collective nonaffine displacements in amorphous materials during large-amplitude oscillatory shear, Phys. Rev. E 95 (2017) 023002.

[33] N.V. Priezjev, Molecular dynamics simulations of the mechanical annealing process in metallic glasses: effects of strain amplitude and temperature, J. Non-Cryst. Solids 479 (2018) 42.

[34] N.V. Priezjev, The yielding transition in periodically sheared binary glasses at finite temperature, Comput. Mater. Sci. 150 (2018) 162.

[35] N.V. Priezjev, Slow relaxation dynamics in binary glasses during stress-controlled tension-compression cyclic loading, Comput. Mater. Sci. 153 (2018) 235.

[36] A.D.S. Parmar, S. Kumar, S. Sastry, Strain localization above the yielding point in cyclically deformed glasses, Phys. Rev. X 9 (2019) 021018.

[37] N.V. Priezjev, M.A. Makeev, The influence of periodic shear on structural relaxation and pore redistribution in binary glasses, J. Non-Cryst. Solids 506 (2019) 14.

[38] N.V. Priezjev, M.A. Makeev, Structural transformations during periodic deformation of low-porosity amorphous materials, Modell. Simul. Mater. Sci. Eng. 27 (2019) 025004

[39] N.V. Priezjev, Accelerated relaxation in disordered solids under cyclic loading with alternating shear orientation, J. Non-Cryst. Solids 525 (2019) 119683.

[40] N.V. Priezjev, Shear band formation in amorphous materials under oscillatory shear deformation, Metals 10 (2020) 300.
[41] P.K. Jana, N.V. Priezjev, Structural relaxation in amorphous materials under cyclic tension-compression loading, J. Non-Cryst. Solids 540 (2020) 120098.

[42] H. Li, H. Liu, H. Peng, Atomic dynamics under oscillatory shear in metallic glasses, J. Non-Cryst. Solids 539 (2020) 120069.

[43] N.V. Priezjev, Alternating shear orientation during cyclic loading facilitates yielding in amorphous materials, J. Mater. Eng. Perform. 29 (2020) 7328.

[44] G.-J. Lyu, J.-C. Qiao, Y. Yao, J.-M. Pelletier, D. Rodney, J. Morthomas, C. Fusco, Dynamic correspondence principle in the viscoelasticity of metallic glasses, Scr. Mater. 174 (2020) 39.

[45] N.V. Priezjev, A delayed yielding transition in mechanically annealed binary glasses at finite temperature, J. Non-Cryst. Solids 548 (2020) 120324.

[46] W. Kob, H.C. Andersen, Testing mode-coupling theory for a supercooled binary Lennard-Jones mixture: the van hove correlation function, Phys. Rev. E 51 (1995) 4626.

[47] T.A. Weber, F.H. Stillinger, Local order and structural transitions in amorphous metal-metalloid alloys, Phys. Rev. B 31 (1985) 1954.

[48] S.J. Plimpton, Fast parallel algorithms for short-range molecular dynamics, J. Comp. Phys. 117 (1995) 1.

[49] M.P. Allen, D.J. Tildesley, Computer Simulation of Liquids, Clarendon, Oxford, 1987.

[50] M. Utz, P.G. Debenedetti, F.H. Stillinger, Atomistic simulation of aging and rejuvenation in glasses, Phys. Rev. Lett. 84 (2000) 1471.

[51] D.J. Lacks, M.J. Osborne, Energy landscape picture of overaging and rejuvenation in a sheared glass, Phys. Rev. Lett. 93 (2004) 255501.

[52] M.L. Falk, J.S. Langer, Dynamics of viscoplastic deformation in amorphous solids, Phys. Rev. E 57 (1998) 7192.

[53] J. Ding, Y.Q. Cheng, E. Ma, Correlating local structure with inhomogeneous elastic deformation in a metallic glass, Appl. Phys. Lett. 101 (2012) 121917.

[54] B.S. Shang, M.Z. Li, Y.G. Yao, Y.J. Lu, W.H. Wang, Evolution of atomic rearrangements in deformation in metallic glasses, Phys. Rev, E 90 (2014) 042303.

[55] N.V. Priezjev, The effect of thermal history on the atomic structure and mechanical properties of amorphous alloys, Comput. Mater. Sci. 174 (2020) 109477.

[56] N.V. Priezjev, Spatiotemporal analysis of nonaffine displacements in disordered solids sheared across the yielding point, Metall. Mater. Trans. A 51 (2020) 3713.

[57] M. Singh, M. Ozawa, L. Berthier, Brittle yielding of amorphous solids at finite shear rates, Phys. Rev. Mater. 4 (2020) 025603.

[58] N.V. Priezjev, The effect of cryogenic thermal cycling on aging, rejuvenation and mechanical properties of metallic glasses, J. Non-Cryst. Solids 503 (2019) 131.

[59] Q.-L. Liu, N.V. Priezjev, The influence of complex thermal treatment on mechanical properties of amorphous materials, Comput. Mater. Sci. 161 (2019) 93.

[60] N.V. Priezjev, Potential energy states and mechanical properties of thermally cycled binary glasses, J. Mater. Res. 34 (2019) 2664.

[61] T.S. Ingebrigtsen, J.C. Dyre, T.B. Schroder, C.P. Royall, Crystallization instability in glass-forming mixtures, Phys. Rev. X 9 (2019) 031016.

[62] B. Deng, Y. Shi, On measuring the fracture energy of model metallic glasses, J. Appl. Phys. 124 (2018) 035101. 